\title{
Digestive Diseases
}

Allocation 164

Amyloidosis 170

Appendicitis 76

Asia 130

Balloon-assisted enteroscopy 63

Barcelona Clinic Liver Cancer system 95

Barrett's esophagus 10,17

Biologicals 83

Budesonide 155

Chemoradiotherapy 30

Cholangiocarcinoma 118, 126, 130

Cholestasis 152

Chronic hepatitis B infection 138

- pancreatitis 43

Cirrhosis 112

Cochrane reviews 43

Coeliac disease 57

Combination 104

Crohn's disease 83

Cyclosporine 91

Direct role of viral proteins 138

- viral factors in hepatocarcinogenesis 138

Diverticulitis 76

Double-balloon enteroscopy 63

Dysphagia 6

Endoscopic resection 17

- therapy 10, 43

- ultrasound 126
Enzyme therapy 43

Eosinophilic esophagitis 6

Esophageal adenocarcinoma 10

- cancer 21, 30

- resection 21

Extended indication 51

Familial amyloid polyneuropathy 170

First-pass effect 155

Fluorescence in situ hybridization 126

Food impaction 6

Gallbladder cancer 118

Gastroesophageal reflux disease 6, 10

Gluten-free diet 57

Guidelines 43

Hepatocarcinogenesis 138

Hepatocellular carcinoma 95, 104, 130, 138

High-grade intraepithelial neoplasia 17

Immunosuppression 83,155

Indication 164

Inflammatory bowel disease 91

Laparoscopic appendectomy 76

Liver cancer 138

- transplantation 112, 164

MALT lymphoma 38

Metaplasia 10

Molecularly targeted therapy 104

Mucosal healing 83
Neuropathy 170

Noncoeliac gluten sensitivity 57

Open appendectomy 76

Outcome 51, 164

Pancreatic cancer 51

Primary biliary cirrhosis 152

- sclerosing cholangitis 118,152

Prognosis 95

Recurrence 155

Resection 51

Single-balloon enteroscopy 63

Small bowel endoscopy 63

Sorafenib 104

Spiral enteroscopy 63

Squamous cell carcinoma 30

Staging systems 95

Step-up approach 83

Surgery 21, 38

Surgical resection 112

Surveillance in primary sclerosing cholangitis 118

Top-down approach 83

Transplantation 155

Treatment 38

Ulcerative colitis 91 